\title{
Evaluation of OAR dose sparing and plan robustness of beam-specific PTV in lung cancer IMRT treatment
}

Yu Chang ${ }^{1 \dagger}$, Feng Xiao ${ }^{2 \dagger}$, Hong Quan ${ }^{2 *}$ and Zhiyong Yang ${ }^{1 *}$ (D)

\begin{abstract}
Purpose: Margins are employed in radiotherapy treatment planning to mitigate the dosimetric effects of geometric uncertainties for the clinical target volume (CTV). Here, we proposed a margin concept that takes into consideration the beam direction, thereby generating a beam-specific planning target volume (BSPTV) on a beam entrance view. The total merged BSPTV was considered a target for optimization. We investigated the impact of this novel approach for lung intensity-modulated radiotherapy (IMRT) treatment, and compared the treatment plans generated using BSPTV with general PTV.

Methods and materials: We generated the BSPTV by expanding the CTV perpendicularly to the incident beam direction using the $2 \mathrm{D}$ version of van Herk's margin concept. The BSPTV and general PTV margin were analyzed using digital phantom simulation. Fifteen lung cancer patients were used in the planning study. First, all patient targets were performed with the CTV projection area analysis to select the suitable beam angles. Then, BSPTV was generated according to the selected beam angles. IMRT plans were optimized with the general PTV and BSPTV as the target volumes, respectively. The dosimetry metrics were calculated and evaluated between these two plans. The plan robustness of both plans for setup uncertainties was evaluated using worst-case analysis.

Results: Both general PTV and BSPTV plans satisfied the CTV coverage. In addition, the BSPTV plans improved the sparing of high doses to target-surrounding lung tissues compared to the general PTV plans. Both $D_{\text {mean }}$ of Ring PTV and Ring BSPTV were significantly lower in BSPTV plans (38.89 Gy and $39.43 \mathrm{~Gy}$ ) compared to the general PTV plans (40.27 Gy and 40.68 Gy). The V20, V5, and mean lung dose of the affected lung were significant lower in BSPTV plans (16.20\%, 28.75\% and $8.93 \mathrm{~Gy})$ compared to general PTV plans (16.69\%, 29.22\% and 9.18 Gy). In uncertainty scenarios, about $80 \%$ of target coverage was achieved for both general PTV and BSPTV plans.
\end{abstract}

Conclusions: The results suggested that plan robustness can be guaranteed in both the BSPTV and general PTV plans. However, the BSPTV plan spared normal tissues, such as the lungs, significantly better compared to the general PTV plans.

Keywords: OAR sparing, Plan robustness, Van Herk's margin concept, Beam specific PTV

*Correspondence: csp6606@sina.com; 284592664@qq.com

${ }^{\dagger}$ Yu Chang and Feng Xiao have contributed equally to this work

${ }^{1}$ Cancer Center, Union Hospital, Tongji Medical College, Huazhong

University of Science and Technology, Wuhan 430022, China

${ }^{2}$ Department of Medical Physics, School of Physics and Technology,

Wuhan University, Wuhan 430072, China

\begin{abstract}
Introduction
Intensity-modulated radiotherapy (IMRT) can deliver conformal dose distributions to tumors. However, the inter-fractional uncertainties during treatment results in deviations between the planned and actual dose distributions [1-3]. These uncertainties can result in underdosing
\end{abstract} original author(s) and the source, provide a link to the Creative Commons licence, and indicate if changes were made. The images or other third party material in this article are included in the article's Creative Commons licence, unless indicated otherwise in a credit line to the material. If material is not included in the article's Creative Commons licence and your intended use is not permitted by statutory regulation or exceeds the permitted use, you will need to obtain permission directly from the copyright holder. To view a copy of this licence, visit http://creativecommons.org/licenses/by/4.0/. The Creative Commons Public Domain Dedication waiver (http://creativeco mmons.org/publicdomain/zero/1.0/) applies to the data made available in this article, unless otherwise stated in a credit line to the data. 
of the clinical target volume (CTV), or overdosing of organs at risk (OARs) [1].

Current clinical practice accounts for uncertainties by using a safety margin that is defined as the planning target volume (PTV) [4-6]. To achieve a higher probability of the CTV coverage under uncertainties, a larger margin for PTV is needed. However, this can result in a higher dose being delivered to surrounding normal tissues [7]. In the van Herk's PTV recipe, uncertainties are separated into two types: systematic and random uncertainties [4]. Systematic uncertainties affect all treatment fractions in the same way, but vary stochastically across the patient population. They can be modeled as displacements of the CTV relative to the blurred dose distribution. Random uncertainties vary from treatment to treatment, and can be modeled as blurring of the cumulative dose distribution.

Geometric uncertainties, including both systematic and random uncertainties, might not simply blur the cumulative dose distribution isotropically. In reality, photon beam radiation deposits exponentially attenuation dose with depth, and whilst the lateral fall-off is much sharper $[1,6]$. In other words, small displacements in beam direction result in small deviations from the planned dose, whereas displacements perpendicular to beam direction can result in severe underdosing due to the target moving out of the beam penumbra [4]. Therefore, it is physically impossible to generate a dose distribution in photon radiation with a negligible dose outside the PTV due to the low dose bath in the beam direction.

The per-beam margin concept in the proton radiation was first proposed by Peter Park et al. [8]. In their study, this beam specific PTV concept was first used to account for the setup and range uncertainties in the prostate and thoracic sites of the proton radiotherapy. Based on their study, we modified the van Herk's margin method, generating margins that vary with the beams' incident directions, thereby only accounting for uncertainties perpendicular to beam incident directions.

Tsang et al. [9] proposed modifications to the van Herk's margin concept by considering margins on the perpendicular direction of each beam in prostate cancer radiotherapy. Tsang et al. [10] also used an adapted beam dependent margin concept, which combined the beam dependent margin and probabilistic planning optimization together to optimize the trade-off between the target coverage and the surrounding rectum and bladder sparing. Their results showed that using the adapted beam dependent margin, better OAR dose sparing could be achieved compared to the general margin. However, in their study, the plan robustness between the general PTV optimization plan and the beam dependent PTV optimization plan was not compared.
Lung cancer radiation treatment is subject to respiratory and setup uncertainties. The therapeutic ratio in lung cancer is essential to ensure adequate coverage of the moving target volume while sparing surrounding normal tissues. In this study, we focused on generating margins that were dependent on the beams' incident directions, and merged them as a beam-specific PTV (BSPTV) in lung cancer radiotherapy. Subsequently, we compared the dose distributions between the general PTV and BSPTV optimized IMRT plans, and evaluated the plan robustness for the setup uncertainties between these two optimization strategy plans.

\section{Materials and methods}

Patient cohort

For this retrospective study, a total of 15 lung cancer patients who underwent IMRT between September 2018 and December 2018 were selected. All patients were enrolled in an institutional review board-approved retrospective data collection protocol, and completed lung radiotherapy treatment.

Patients were acquired 4DCT using a Big Bore CT simulator (Brilliance, Philips Healthcare, Cleveland, OH, USA) with a real-time position management system (RPM, Varian Medical Systems, Palo Alto, CA, USA). The gross tumor volume (GTV) and CTV was contoured on average-weighted CT images. To simplify our analysis, only tumors in one side of lungs were selected. In addition, all OARs, such as lungs and spinal cord were contoured on average-weighted CT images. The averageweighted CT images were used for plan optimization and dose calculation.

\section{Beam-specific PTV concept}

To generate the BSPTV for photon radiotherapy, we expanded the CTV perpendicularly to each incident beam direction using the 2D version of van Herk's margin concept. We chose not to add margins in the CTV in the incident beam direction, because the percentage depth dose reduction in the incident beam direction was very small. We obtained the final BSPTV by merging each beam expansions. Figure 1 shows the simulation of target coverage differences between the target moving parallel to beam direction and perpendicular to beam direction. Figure 2 shows the geometric differences between the original PTV and the BSPTV for the same CTV in the axial slice.

The setup inter-fractional uncertainties considered in this study were defined in the patient's left-right, anterior-posterior and superior-inferior directions, and were assumed to be normally distributed with no correlations between them. The projection of the 3D Gaussian distribution, in beam direction, into a 2D Gaussian 


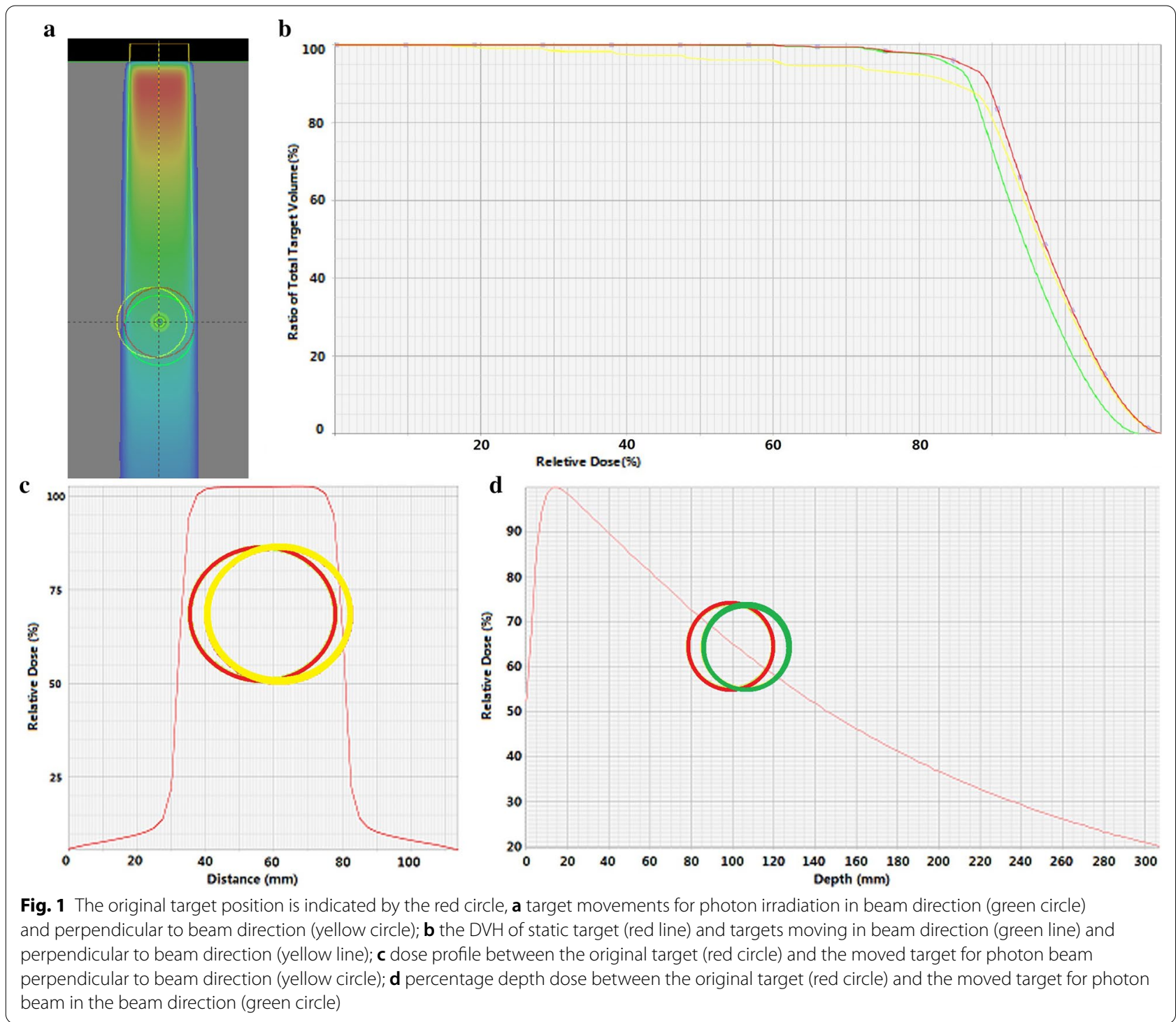

distribution was defined using the van Herk's margin concept $[4,9,11]$

$$
M=\alpha \Sigma+\beta \sqrt{\sigma^{2}+\sigma_{p}^{2}}-\beta \sigma_{p}
$$

Variables $\Sigma$, $\sigma$, and $\sigma_{\mathrm{p}}$ are two-dimensional column vectors for the directions perpendicular to the incident beam angle, thereby representing the systematic uncertainties, random uncertainties and the beam penumbra, defined as the distance between the 20 and $80 \%$ isodose levels, respectively. In this study, the beam penumbras $\left(\sigma_{p}\right)$ was $3.2 \mathrm{~mm}$ in water. The coefficients $\alpha$ and $\beta$, which depend on the intended probability of target dose coverage, were calculated by solving the closed-formed dose population histogram, following the integral formula in
Appendix 2 of a previous study [4]. Our method to calculate the $2 \mathrm{D}$ margin from the direction perpendicular to each beam was implemented as a standalone MATLAB (MathWorks, Natick, MA, USA) program. To ensure that $90 \%$ of the patients received at least $95 \%$ of the prescribed dose across the whole of the target, in our programmed $2 \mathrm{D}$ margin script, the corresponding coefficients were $\alpha=2.15$ and $\beta=1.64$. In addition, we set the systematic uncertainties to $\Sigma=2 \mathrm{~mm}$, and random uncertainties to $\sigma=2 \mathrm{~mm}$ according to the previous study and our clinical results $[4,12]$, the Margin $(M) \approx 4.4 \mathrm{~mm}$. The automatic generated 2D margins of each beam were imported into Eclipse TPS (Varian Medical Systems, Palo Alto, CA, USA) and merged as the BSPTV for subsequent optimization. 

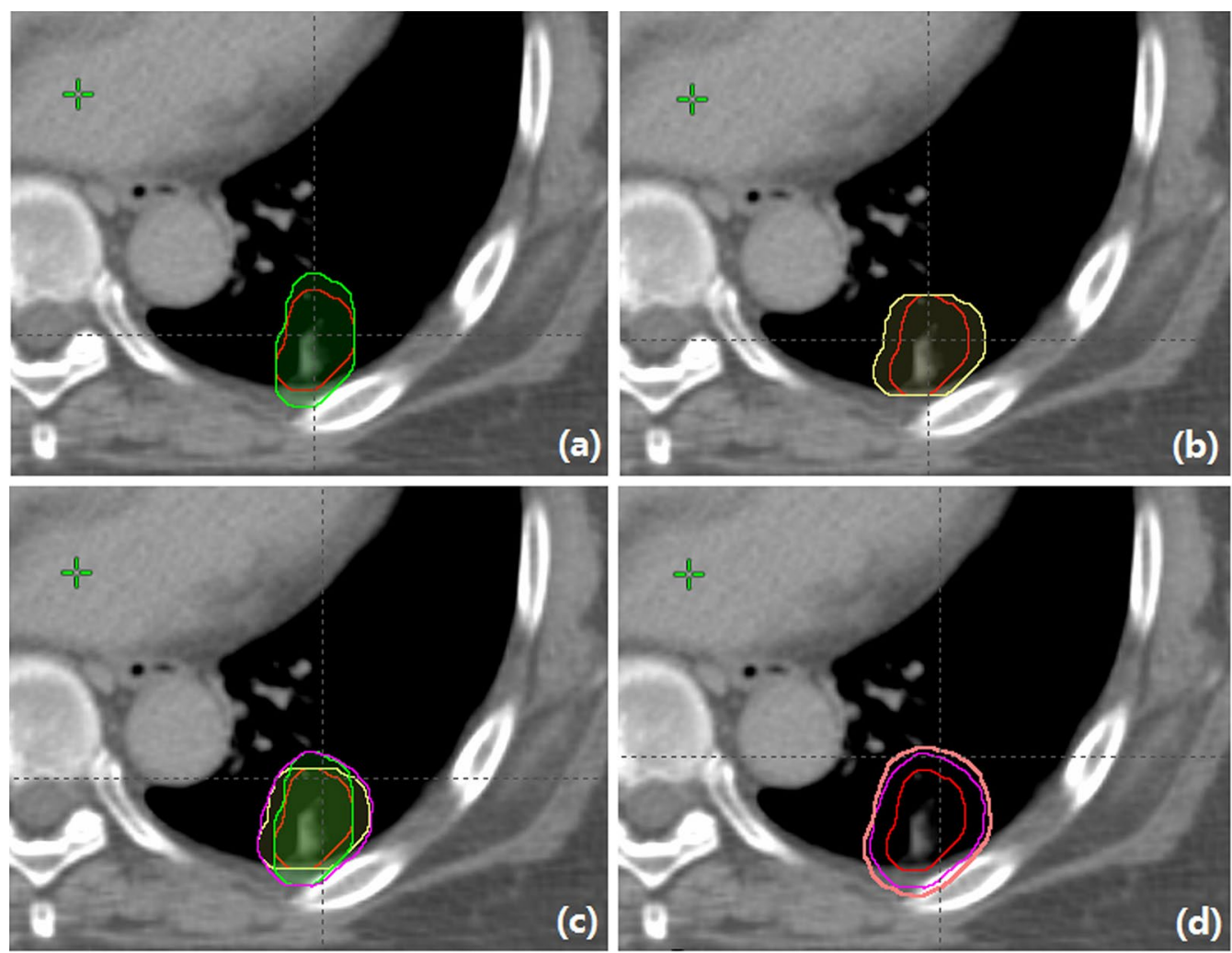

Fig. 2 The isocenter axial slice of the CTV (red line) expansion generated using 2D VHMR for $90^{\circ}$ beam (green line) in a and $180^{\circ}$ beam (yellow line) in $\mathbf{b}$; c shows the isocenter axial slices of the union BSPTV for all beams (pink line); $\mathbf{d}$ shows the isocenter axial slices of the union BSPTV (pink line) and general PTV (light brown line).

The general PTV was margined by the 3D van Herk's margin concept, with the same systematic and random uncertainties as the BSPTV as follows: the systematic uncertainties $\Sigma=2 \mathrm{~mm}$, random uncertainties $\sigma=2 \mathrm{~mm}$, $\alpha=2.5, \beta=1.64$, and $M \approx 5 \mathrm{~mm}$.

\section{Phantom simulation}

A digital water phantom simulation was used to evaluate the conformity of the dose distribution to the OAR sparing of these two margin concepts. The influence of the number of beams to the volume of the BSPTV was also evaluated using this digital water phantom.

In this simulation model, the water phantom was $40 \times 40 \times 40 \mathrm{~cm}^{3}$ with a spherical CTV of $4 \mathrm{~cm}$ diameter in the center, which roughly corresponded to the average CTV sizes of our patient data. The dose grid resolution for the dose calculation was $2.5 \mathrm{~mm}$. Two types of $50 \mathrm{~Gy} / 25 \mathrm{~F}$ treatment plans were designed for both general PTV and BSPTV, and the generated dose distributions were such that $98 \%$ of the PTV received $100 \%$ of the prescribed dose. The general PTV margin $(\mathrm{M} \approx 5 \mathrm{~mm})$ was applied around the CTV for the plans with general PTV as the target volume. The BSPTV margin $\left(\mathrm{M} \approx 4.4 \mathrm{~mm}\right.$, in $0^{\circ}, 30^{\circ}, 60^{\circ}$ and $90^{\circ}$ directions $)$ was applied around the CTV for plans with the BSPTV as a target volume. The first type involved two clinical IMRT plans using 4 coplanar beams with $0^{\circ}, 30^{\circ}, 60^{\circ}$ and $90^{\circ}$ directions with the general PTV and BSPTV as the target volume, respectively. The plan conformity index (CI, $\left.\mathrm{CI}=100 \% \times\left[\mathrm{TV}_{\mathrm{PI}}\right]^{2} /\left[\mathrm{PI}_{100} \times \mathrm{TV}\right]\right)$ was optimized to keep the $\mathrm{CI}$ in both general PTV and BSPTV plans above $80 \%$. $\mathrm{TV}$ represented the target volume, $\mathrm{TV}_{\mathrm{PI}}$ represented the volume of the target covered by the prescribed isodose, and $\mathrm{PI}_{100}$ represented the volume receiving $100 \%$ of the prescribed isodose. The conformity was better as the index approached $100 \%$. The second type involved hypothetical plans with an ideal dose distribution (such as a VMAT plan with a spherically symmetric dose that falls off in all directions), resulting in a CI above $90 \%$.

Three plans were generated to evaluate the influence of the number of beams to the volume of the BSPTV. The three plans were generated using three, five, or seven coplanar beams with equal angle intervals. Subsequently, the volume difference between the general PTV and BSPTV of the same spherical CTV of each plan was calculated. 


\section{CTV projection area analysis}

In this study, the mathematical relationship between the margin volume and the projection area of the target was analyzed. The calculation and analysis details are shown in "Appendix". Based on a patient's CT data set, we calculated the CTV projection area in a beam direction from $0^{\circ}$ to $359^{\circ}$. Because the BSPTV of a beam is a $2 \mathrm{D}$ margin of the CTV in the beam direction, the projection area of the CTV in the beam direction is an index that can be used to evaluate the volume of $2 \mathrm{D}$ expansion of the CTV. The projection area of the CTV was calculated for a full circle of beam angles (0-359) at increments of 1 to yield a patient-specific CTV projection area curve as a function of the beam angle. For example, Fig. 3a shows the CTV projection area of one patient with respect to the beam angle.

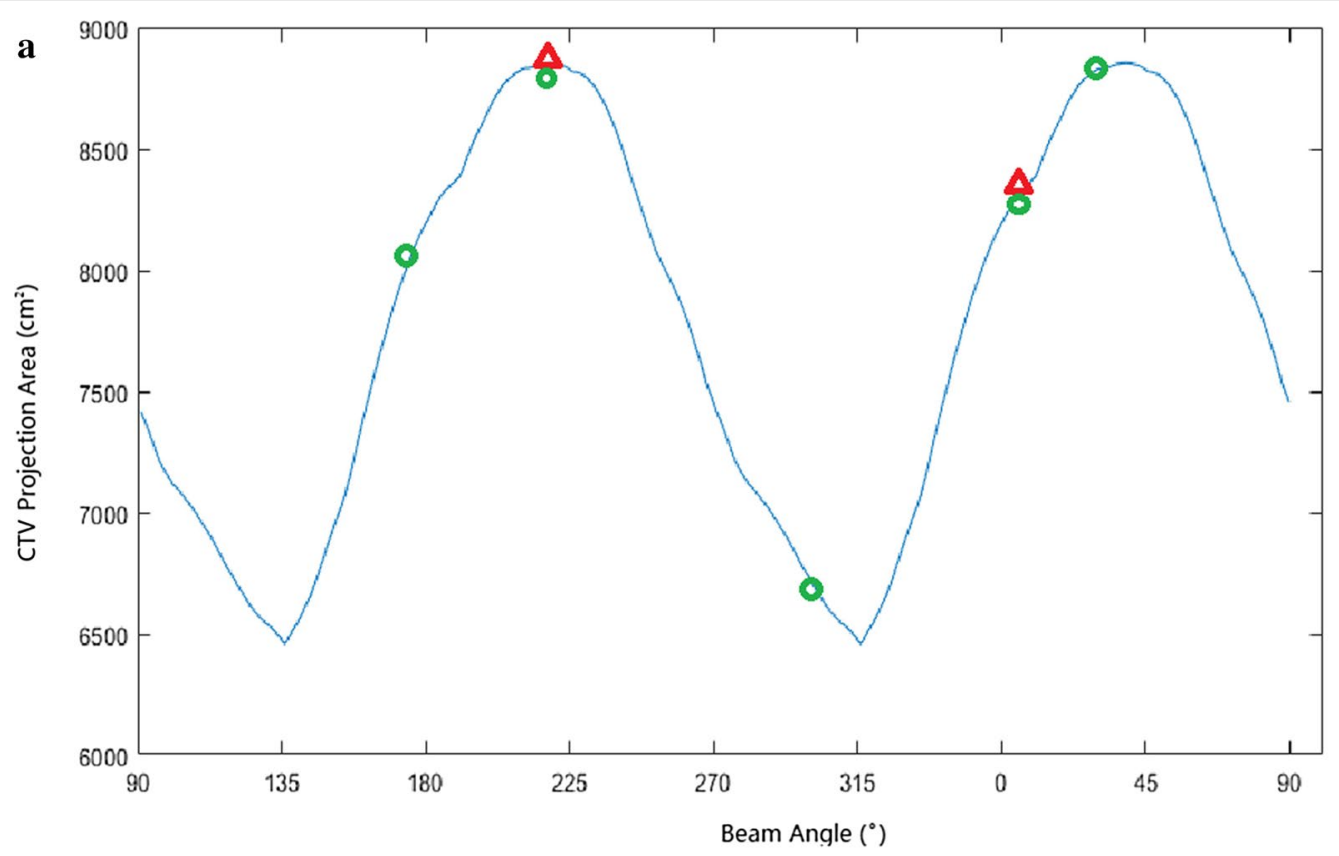

b

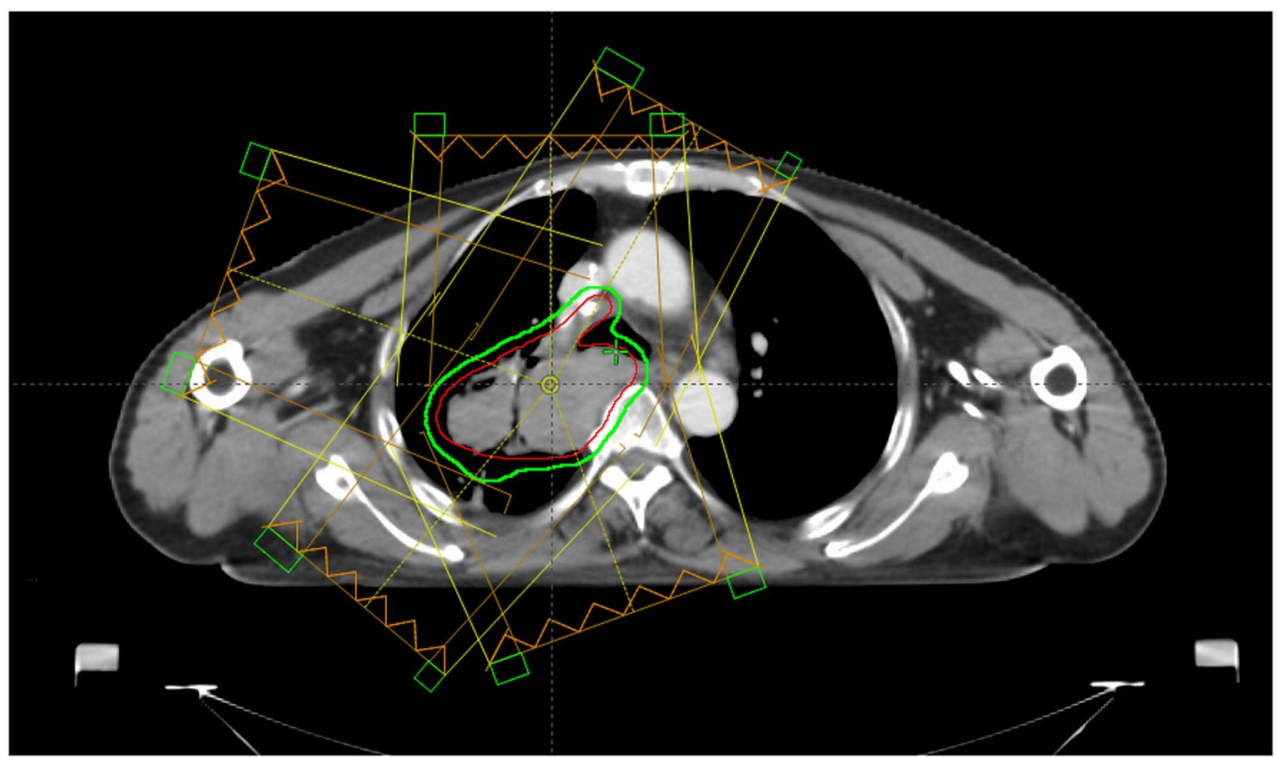

Fig. 3 The example case's CTV projection area with respect to the beam angle is shown in $\mathbf{a}$. The selected beam angles $\left(0^{\circ}, 30^{\circ}, 160^{\circ}, 220^{\circ}\right.$, and $290^{\circ}$ ) are indicated as green circles. The first and second maximum projection beam angles are indicated as red triangles; the axial slice view of the example case's beams are shown in $\mathbf{b}$ 


\section{IMRT planning}

IMRT plans were optimized by the Eclipse v13.6 treatment planning system (Varian Medical System, Palo Alto, CA, USA) and simulated with 6 MV Xray of Trilogy linac (Varian Medical System, Palo Alto, CA, USA). The plan final dose was calculated using the Acuros algorithm and prescribed to $60 \mathrm{~Gy}$ in 30 fractions. First, we selected 4-5 beam angles according to the CTV projection area curve to make sure that the projection area on the YOZ plane had higher values than other beam angles. To avoid the beam penetrating both lungs, most beams incidents were set in the AP directions. Second, the general PTV and BSPTV margins were generated as the description in Beam-specific PTV concept section. Next, two plan optimization strategies were designed to compare the dose distribution differences between the general PTV and BSPTV. The optimization objective for all plans was to first achieve $100 \%$ of the prescribed dose to the target volume, then to minimize the dose to OARs. We first optimized all patients' plans on the target volume of the general PTV. Then, all optimization objective parameters were kept unchanged, a new plan was created, and only the target volume was changed to BSPTV and this BSPTV plan was re-optimized. After the optimization of both plans was completed, all plans were normalized to facilitate dose comparisons. The normalization point was the target $D_{98 \%}$ of $60 \mathrm{~Gy}$, where $\mathrm{D}_{\mathrm{x} \%}$ was defined as the lowest dose covering $x \%$ of the volume. To exclude the influence of the conformity of the dose distribution to the plan evaluation, plans with a CI below $80 \%$ were re-optimized until the CI was above $80 \%$.

\section{Plan evaluation}

To quantify the differences between the general PTV and BSPTV plans, dose-volume histograms were used to assess the dose coverage and conformity of targets and the protection of OARs. The target evaluation parameters were D98\% (target coverage), D2\%, CI, and homogeneity index (HI) [13]. The HI, defined as $100 \% \times\left(\mathrm{D}_{2 \%}-\mathrm{D}_{98 \%}\right) /$ $\mathrm{D}_{50 \%}$, was used to evaluate dose homogeneity within each target volume. Plans that are more homogenous, have $\mathrm{HI}$ values that are closer to $0 \%$ [13]. V5, V20, and mean doses to both lungs were compared. For the spinal cord, the $\mathrm{D}_{1 \%}$ was compared. To evaluation the adjacent normal tissue of CTV, a 2-cm ring of the CTV was margined as the CTV margin. The Ring PTV or Ring BSPTV were created by the CTV margin with the general PTV or BSPTV being subtract. The volume and mean doses of Ring PTV and Ring BSPTV were compared.

\section{Robust analysis}

Over the course of radiation therapy, interfractional uncertainties occur between treatment fractions, such as set-up uncertainties and anatomical variations. In this study, we evaluated the set-up uncertainties using the uncertainty dose evaluation of Eclipse treatment planning system, assuming inter-fractional setup uncertainties of $\pm 5 \mathrm{~mm}$ in AP, LR and SI directions, total 6 scenarios. The van Herk margin recipe was designed for $90 \%$ of coverage of the patients with a minimal dose of $95-100 \%$ of the target volume, therefore, the ratio of scenarios that satisfied the clinical specification that the $100 \%$ of CTV above the $95 \%$ prescription dose was evaluated.

\section{Statistics analysis}

SPSS 24.0 software (IBM, Armonk, NY, USA) was used for statistical analyses of all dosimetric metrics. We conducted a paired, two-tailed Wilcoxon signed-rank test to compare the dose distributions between general PTV and BSPTV plans. $P<0.05$ was considered statistically significant.

\section{Results \\ Phantom simulation}

Table 1 shows the phantom simulation results of the 4-field IMRT plans and the hypothetical plans with an ideal dose distribution. For the plans with general PTV set as the target volume, the CI of the 4-field IMRT plans was $85.18 \%$ and the CI of the hypothetical plans with an ideal dose distribution was $92.80 \%$. The mean value of the Ring PTV was 53.08 Gy in 4-field IMRT plans, and 53.34 in the hypothetical plans with an ideal dose distribution. For plans with BSPTV set as the target volume, the CI of 4-field IMRT plans was $83.25 \%$ and the CI of the hypothetical plans with an ideal dose distribution was $91.92 \%$. The mean value of the Ring PTV was 52.89 Gy in 4-field IMRT plans, and 52.97 in the hypothetical plans with an ideal dose distribution. Both 4-field IMRT plans and hypothetical plans with an ideal dose distribution were $100 \%$ satisfied the clinical specification of plan robustness, as shown in Table 2.

The volume difference between the general PTV and BSPTV of the same spherical CTV was $-9.06 \%$ in the three beams plan, $-7.11 \%$ in the five beams plan, and $-5.41 \%$ in the seven beams plan.

\section{CTV projection area analysis}

For different patients, the shape of the CTV projection area curve varied. The results of our mathematical and geometrical analysis ("Appendix") suggested that the smallest BSPTV was obtained with the beam irradiated perpendicular to the maximum average length of the target in the XOY plane. Table 3 lists the beam angles that correspond to the first and second maximum values of the CTV projection area for each patient. 
Table 1 Summary of dose to targets and OARs in water phantom simulation

\begin{tabular}{|c|c|c|c|c|}
\hline Parameter & $\begin{array}{l}\text { General PTV 4-field IMRT } \\
\text { plan }\end{array}$ & $\begin{array}{l}\text { BSPTV 4-field IMRT } \\
\text { plan }\end{array}$ & $\begin{array}{l}\text { General PTV ideal dose } \\
\text { distribution plan }\end{array}$ & $\begin{array}{l}\text { BSPTV ideal dose } \\
\text { distribution plan }\end{array}$ \\
\hline CTV, $D_{98 \%}$ & 62.5 & 62.5 & 61.5 & 61.0 \\
\hline General PTV, volume $\left(\mathrm{cm}^{3}\right)$ & 65.9 & 65.9 & 65.9 & 65.9 \\
\hline General PTV, D98\% (Gy) & 60.0 & 59.9 & 60.0 & 59.5 \\
\hline General PTV, D $2 \%$ (Gy) & 64.5 & 64.6 & 63.3 & 63.4 \\
\hline General PTV, CI (\%) & 85.2 & 85.1 & 92.8 & 91.1 \\
\hline General PTV, HI (\%) & 3.5 & 3.6 & 3.4 & 3.4 \\
\hline BSPTV, volume $\left(\mathrm{cm}^{3}\right)$ & 62.5 & 62.5 & 62.5 & 62.5 \\
\hline BSPTV, $D_{98 \%}(G y)$ & 60.1 & 60.0 & 60.2 & 60.0 \\
\hline BSPTV, $D_{2 \%}(G y)$ & 64.5 & 64.6 & 63.3 & 63.4 \\
\hline BSPTV, Cl & 82.4 & 83.3 & 90.7 & 91.9 \\
\hline BSPTV, HI & 3.5 & 3.6 & 3.4 & 3.4 \\
\hline Ring PTV, $D_{\text {mean }}(G y)$ & 53.1 & 52.4 & 53.3 & 52.6 \\
\hline Ring BSPTV, $D_{\text {mean }}(G y)$ & 53.5 & 52.9 & 53.7 & 53.0 \\
\hline Ring PTV, volume $\left(\mathrm{cm}^{3}\right)$ & 61.2 & 61.2 & 61.2 & 61.2 \\
\hline Ring BSPTV, volume $\left(\mathrm{cm}^{3}\right)$ & 64.6 & 64.6 & 64.6 & 64.6 \\
\hline
\end{tabular}

$\mathrm{HI}$ homogeneity index, $\mathrm{Cl}$ conformity index

Table 2 The ratios of scenarios satisfied the clinical specifications that the $100 \%$ target volume being above the $100 \%$ or $95 \%$ prescription dose in water phantom simulation

\begin{tabular}{|c|c|c|c|c|}
\hline \multirow{2}{*}{$\begin{array}{l}\text { Plan } \\
\text { name }\end{array}$} & \multicolumn{2}{|c|}{ General PTV plan (\%) } & \multicolumn{2}{|c|}{ BSPTV plan (\%) } \\
\hline & $\begin{array}{l}100 \% \\
\text { prescription } \\
\text { dose }\end{array}$ & $\begin{array}{l}95 \% \\
\text { prescription } \\
\text { dose }\end{array}$ & $\begin{array}{l}100 \% \\
\text { prescription } \\
\text { dose }\end{array}$ & $\begin{array}{l}95 \% \\
\text { prescription } \\
\text { dose }\end{array}$ \\
\hline $\begin{array}{l}\text { 4-field } \\
\text { IMRT } \\
\text { plan }\end{array}$ & 67 & 100 & 67 & 100 \\
\hline $\begin{array}{l}\text { Ideal } \\
\text { dose } \\
\text { distri- } \\
\text { bution } \\
\text { plan }\end{array}$ & 67 & 100 & 67 & 100 \\
\hline
\end{tabular}

Volume difference between the general PTV and BSPTV

Beams were selected according to the CTV projection area curve. A larger CTV projection area indicated a smaller $2 \mathrm{D}$ expansion of the CTV and more sparing of the adjacent OARs. The beam angles of an example case are presented in Fig. 3b. Table 3 lists the volume of the general PTVs and BSPTVs for all patients. For all patients, the mean (SD) volume reduction of the BSPTV compared to the general PTV was $-10.27 \%$ (7.11\%).
Dose difference between the general PTV optimization plan and BSPTV optimization plan

Table 4 shows the general PTV and BSPTV $\mathrm{D}_{98 \%}, \mathrm{D}_{2 \%}, \mathrm{CI}$, and $\mathrm{HI}$ for the two plans. For all patients, the $\mathrm{D}_{2 \%}$ did not significantly differ between the general PTV and BSPTV plans. In addition, the CI of the general PTV in the general PTV optimization plan and the CI of the BSPTV in the BSPTV optimization plan were not significantly different $(p=0.281)$. The CI of the BSPTV was higher in the BSPTV optimization plan compared to the general PTV plan $(p=0.003)$. In both general PTV plans, the $\mathrm{D}_{98 \%}$ of the BSPTV was higher compared to that of the general $\operatorname{PTV}(p=0.003)$ and BSPTV plans $(p=0.001)$.

The volume of the Ring PTV was smaller than the volume of the Ring BSPTV $(p=0.001)$. The BSPTV plans had significantly lower mean dose for Ring PTV and Ring BSPTV compared to the general PTV plan $(p=0.001$ and $p=0.001)$. Furthermore, compared with the general PTV plans, the BSPTV plans showed a decrease in the V5 and mean dose of the affected lung ( $p=0.013$, $0.005)$, contralateral lung $(p=0.028,0.012)$, and total lungs $(p=0.015,0.003)$ and a decrease in the V20 of the affected lung $(p=0.005)$ and total lungs $(p=0.008)$.

\section{Robust analysis}

Table 5 shows the results of the robust analysis for both general PTV plans and BSPTV plans. The ratios of scenarios showed that $100 \%$ of the CTV was still above $100 \%$ or $95 \%$ (clinical specification) of the prescription dose calculated for both plans. Moreover, the results showed 
Table 3 Volumes for general PTVs and the union of BSPTVs for all patients, and the beam angles corresponding to the extreme values of CTV projection area and the plan selected beam angles

\begin{tabular}{lcccclc}
\hline Patient nos. & $\begin{array}{l}\text { CTV volume } \\
\left(\mathbf{c m}^{\mathbf{3}}\right)\end{array}$ & $\begin{array}{l}\text { General PTV } \\
\text { volume }\left(\mathbf{c m}^{\mathbf{3}}\right)\end{array}$ & $\begin{array}{l}\text { BSPTV } \\
\text { volume }\left(\mathbf{c m}^{\mathbf{3}}\right)\end{array}$ & $\begin{array}{l}\text { Volume } \\
\text { difference (\%) }\end{array}$ & $\begin{array}{l}\text { Beam angles for maximum, 2nd } \\
\text { maximum ITV projection area }\end{array}$ & $\begin{array}{l}\text { Selected beam angles } \\
1\end{array}$ \\
\hline 36.9 & 83.9 & 76.0 & -10.4 & 204,180 & $90,150,180,230$ \\
2 & 11.5 & 29.2 & 27.9 & -4.7 & 175,210 & $21,150,180,210,335$ \\
3 & 6.7 & 22.9 & 18.8 & -21.8 & 300,320 & $0,30,170,300$ \\
4 & 87.7 & 164.5 & 153.9 & -6.9 & 102,90 & $0,30,160,190,220$ \\
5 & 12.6 & 35.0 & 30.3 & -15.5 & 90,315 & $0,90,175,315$ \\
6 & 3.1 & 12.9 & 11.3 & -14.2 & 195,180 & $70,130,180,210$ \\
7 & 270.9 & 466.2 & 443.6 & -5.1 & 0,220 & $0,30,160,220,290$ \\
8 & 10.2 & 27.6 & 25.6 & -7.8 & 106,320 & $10,40,180,330$ \\
9 & 1.5 & 9.0 & 7.0 & -28.6 & 160,125 & $40,130,180,210$ \\
10 & 57.6 & 155.4 & 146.3 & -6.2 & 45,90 & $40,95,175,225$ \\
11 & 12.3 & 32.9 & 31.3 & -5.0 & 0,210 & $0,130,170,210$ \\
12 & 8.9 & 31.1 & 28.1 & -10.7 & 130,145 & $15,150,180,210,240$ \\
13 & 72.7 & 102.8 & 99.8 & -3.0 & 320,50 & $320,40,0,170$ \\
14 & 9.4 & 26.0 & 24.6 & -5.6 & 270,240 & $240,210,180,150,15$ \\
15 & 14.4 & 40.0 & 36.7 & -8.7 & 250,180 & $210,180,150,120,345$ \\
\hline
\end{tabular}

that the ratios of scenarios of the clinical specifications was not significantly different between the general PTV and BSPTV plans (mean value: 0.966 vs. $0.953, p=0.317$ ). For both general PTV and BSPTV plans, about $80 \%$ of the target coverage could still be achieved in all uncertainty scenarios.

\section{Discussion}

Compared to proton radiotherapy, photon radiotherapy physically cannot produce perfectly conforming dose distributions. There will be a presence of entrance and exit doses due to how photons interact with matter. In this study, we took advantage of the exponential relationship between the absorbed dose and radiological depth. Since small movements in a beam direction result in negligible dose deviation, we designed the BSPTV concept to spare normal tissues in the beam direction. Because of the different shapes of the target in different beam entrance directions, it is important to choose a suitable incidence angle of the beam according to the projection area values. However, surrounding OARs need to be considered when choosing the incidence angle of the beam. Moreover, the distance of the target to the isocenter of the field is related to the utilization efficiency of the radiation, therefore, choosing of the incident angle of the beam is a trade-off between different factors.

In a previous study, it was shown that suboptimal dose conformation could lead to a tighter margin for the target, because the prescription dose escaped out of the target [14]. In our phantom simulation, a better conformity target dose distribution was achieved in the hypothetical plans group (>90\%) compared to the 4-field IMRT plans group (>80\%). However, the plan robustness was not different between the two types of plans. Combined, these results suggested that the conformity of the dose distribution would not have much impact on the plan robustness when a high target conformity $(>80 \%)$ was achieved. In IMRT plans, a high target conformity (>80\%) could easily be achieved, and the plan robustness would not be subjected to the conformity of the dose distribution.

We chose the CTV projection area analysis to identify suitable beam angles to obtain a smaller BSPTV volume and sparing more surrounding lung tissues. The mathematical and geometrical analysis suggested that beams that irradiated perpendicular to the maximum average length of the target in the XOY plane obtained the minimum BSPTV volume. The sparing volume, which represents differences between the general PTV and BSPTV, is dependent on the accumulation of the target projection area in the YOZ plane. Especially for targets with an irregular shape, such as the example case in Fig. 3, the CTV projection area differences between the maximum and the minimum value could be $25 \%$ (Fig. 3a). The CTV projection area curve is symmetric for beam angles with $180^{\circ}$ intervals. Suitable beam angles with a direction close to the tumor can be chosen according to the CTV projection area curve.

The value of the BSPTV is impacted by the beam angles and the number of beams. Table 3 shows that if the number of beams increased from 4 to 5 , the volume differences between the general PTV and BSPTV reduced from 12.50 to $6.94 \%$. The digit phantom simulation of 
Table 4 Summary of dose to targets and OARs for all patients, shown as mean (standard deviation)

\begin{tabular}{|c|c|c|c|}
\hline Parameter & General PTV optimization plan & BSPTV optimization plan & $P^{\mathbf{a}}$ \\
\hline CTV, $D_{98 \%}(G y)$ & $61.68(0.99)$ & $61.40(1.15)$ & 0.280 \\
\hline General PTV, D $D_{98 \%}$ (Gy) & $60.00(0.00)$ & $58.84(1.01)$ & $0.001^{f}$ \\
\hline General PTV, D2\% (Gy) & $63.38(1.35)$ & $63.36(1.53)$ & 0.649 \\
\hline General PTV, Cl & $0.85(0.05)$ & $0.86(0.05)$ & 0.233 \\
\hline General PTV, HI & $0.05(0.02)$ & $0.07(0.03)$ & $0.002^{f}$ \\
\hline BSPTV, $D_{98 \%}(G y)$ & $60.53(0.50)$ & $60.00(0.00)$ & $0.003^{f}, 0.003^{c, f}, 0.001^{d, f}$ \\
\hline BSPTV, $D_{2 \%}(G y)$ & $63.48(1.41)$ & $63.36(1.51)$ & 0.975 \\
\hline BSPTV, $\mathrm{Cl}$ & $0.79(0.07)$ & $0.84(0.05)$ & $0.003^{f}, 0.281^{e}$ \\
\hline BSPTV, HI & $0.05(0.02)$ & $0.05(0.02)$ & 0.100 \\
\hline Ring PTV, $D_{\text {mean }}(G y)$ & $40.27(6.83)$ & $38.89(7.13)$ & $0.001^{f}$ \\
\hline Ring BSPTV, $D_{\text {mean }}(G y)$ & $40.68(6.72)$ & $39.43(7.08)$ & $0.001^{f}$ \\
\hline Ring PTV, volume $\left(\mathrm{cm}^{3}\right)$ & 195.61 (203.99) & & $0.001^{b, f}$ \\
\hline Ring BSPTV, volume $\left(\mathrm{cm}^{3}\right)$ & $201.21(210.89)$ & & \\
\hline Affected lung, V20 & $16.69(11.32)$ & $16.20(10.85)$ & $0.005^{f}$ \\
\hline Contralateral lung, V20 & $0.36(0.01)$ & $0.36(0.01)$ & 1.000 \\
\hline Total lungs, V20 & $7.25(6.40)$ & $7.02(6.00)$ & $0.008^{f}$ \\
\hline Affected lung, V5 & $29.22(14.87)$ & $28.75(14.43)$ & $0.013^{f}$ \\
\hline Contralateral lung, V5 & $6.91(11.71)$ & $6.71(11.56)$ & $0.028^{f}$ \\
\hline Total lungs, V5 & $16.26(12.72)$ & $15.92(12.37)$ & $0.015^{f}$ \\
\hline Affected lung, $D_{\text {mean }}(G y)$ & $9.18(5.51)$ & $8.93(5.28)$ & $0.005^{f}$ \\
\hline Contralateral lung, $D_{\text {mean }}(G y)$ & $0.97(1.43)$ & $0.95(1.42)$ & $0.012^{f}$ \\
\hline Total lungs, $D_{\text {mean }}(G y)$ & $4.79(3.33)$ & $4.67(3.18)$ & $0.003^{f}$ \\
\hline Spinal cord, $D_{1 \%}(G y)$ & $16.14(16.50)$ & $15.72(15.89)$ & $0.009^{f}$ \\
\hline
\end{tabular}

$\mathrm{HI}$ homogeneity index, $\mathrm{Cl}$ conformity index

${ }^{\text {a }}$ Comparison of general PTV optimization plan with BSPTV optimization plan

${ }^{b}$ Comparison of volumes of ring PTV and ring BSPTV

c Comparison of $\mathrm{D}_{98 \%}$ of general PTV and BSPTV in the same general PTV optimization plan

d Comparison of $\mathrm{D}_{98 \%}$ of general PTV and BSPTV in the same BSPTV optimization plan

e Comparison of $\mathrm{Cl}$ of general PTV in the general PTV optimization plan and $\mathrm{Cl}$ of BSPTV in the BSPTV optimization plan

${ }^{f} P<0.05$

plans with three, five, and seven coplanar beams also demonstrated that the volume differences between the general PTV and BSPTV reduced with an increasing number of beams. Taken together, these results suggested that the BSPTV concept is not suitable for treatment with a large number of beams, such as stereotactic body radiation therapy (SBRT), because the BSPTV might be the same with a general PTV.

The clinical specifications of BSPTV plans of cases 5 , 9, and 13 could not be achieved (Table 5). The scenarios, which are not satisfied for the clinical specifications, are all scenarios with set-up uncertainties in SI directions. The reason for the underdosing might be that the target with set-up uncertainties in the SI directions are too close to the beam aperture, therefore, the lateral scattering dose may not be sufficient, especially in lung tissue.

Shusharina et al. [15] proposed a probabilistic clinical target distribution concepts implemented into the probabilistic optimization process in the head and neck cases.
This allowed physicists and physicians to identify the most suitable trade-off between target coverage and sparing of surrounding normal tissues at the treatment planning stage, without having to modify or redraw a CTV. In addition, Witte et al. [16] also tested the probabilistic planning margin volume concepts in the simulated phantom. Watkins et al. [17] defined the definite target volume to deliver extremely high doses to sub-volumes of PTVs in multiple treatment sites. These three studies focused on the influence of the probabilistic dose distribution to the target coverage, and sparing of surrounding normal tissues is another strategy comparing to the BSPTV methods. Since the BSPTV strategy could be applied without modification of the optimization engine of the commercial treatment planning system, it is a much easier and more straightforward strategy.

A considerable amount of work remains to continue to be performed to further improve BSPTV optimization. Both the van Herk's margin concept and our beam 
Table 5 The ratios of scenarios satisfied the clinical specifications that the $100 \%$ target volume being above the $100 \%$ or $95 \%$ prescription dose for all patients

\begin{tabular}{|c|c|c|c|c|}
\hline \multirow{2}{*}{$\begin{array}{l}\text { Patient } \\
\text { nos. }\end{array}$} & \multicolumn{2}{|c|}{ General PTV plan (\%) } & \multicolumn{2}{|c|}{ BSPTV plan (\%) } \\
\hline & $\begin{array}{l}100 \% \\
\text { prescription } \\
\text { dose }\end{array}$ & $\begin{array}{l}95 \% \\
\text { prescription } \\
\text { dose }\end{array}$ & $\begin{array}{l}100 \% \\
\text { prescription } \\
\text { dose }\end{array}$ & $\begin{array}{l}95 \% \\
\text { prescription } \\
\text { dose }\end{array}$ \\
\hline 1 & 50 & 100 & 50 & 100 \\
\hline 2 & 50 & 100 & 50 & 100 \\
\hline 3 & 100 & 100 & 50 & 100 \\
\hline 4 & 100 & 100 & 100 & 100 \\
\hline 5 & 67 & 83 & 50 & 83 \\
\hline 6 & 50 & 100 & 50 & 100 \\
\hline 7 & 100 & 100 & 100 & 100 \\
\hline 8 & 67 & 100 & 67 & 100 \\
\hline 9 & 83 & 100 & 67 & 83 \\
\hline 10 & 100 & 100 & 100 & 100 \\
\hline 11 & 100 & 100 & 83 & 100 \\
\hline 12 & 67 & 100 & 50 & 100 \\
\hline 13 & 17 & 67 & 17 & 67 \\
\hline 14 & 83 & 100 & 83 & 100 \\
\hline 15 & 100 & 100 & 100 & 100 \\
\hline
\end{tabular}

specific margin concept share the following assumptions: the treatment uncertainties follow a Gaussian distribution, which is not appropriate for SBRT treatment, and only rigid translations of the target were accounted for [4]. We will focus on calculating the effects of intra-fraction motion and inter-fractional uncertainties as well as analyzing the rotation and deformation uncertainties of the targets. And we will continue study the BSPTV of two or more targets in one patient, such as primary sites and mediastinal lymph nodes for lung cancer cases.

\section{Conclusion}

Lung tumors are surrounded by normal tissues and OARs, and the low dose delivery to normal tissues needs to be limited. Hence, the IMRT plans with 3-5 beams could effectively control the low dose area in lung treatment. Thus, using BSPTV is highly suitable for the IMRT is plan strategy.

\section{Abbreviations}

CTV: Clinical target volume; PTV: Planning target volume; BSPTV: Beam-specific PTV; IMRT: Intensity modulated radiation therapy; OAR: Organ at risk; DVH: Dose volume histogram; SBRT: Stereotactic body radiation therapy; GTV: Gross tumor volume; SD: Standard deviation.

\section{Acknowledgements}

This work was supported by National Natural Science Foundation of China (Grant No. 81803047) (ZY) and the Fundamental Research Funds for the Central Universities of China (Grant No. 2019kfyXKJC061) (ZY).
Authors' contributions

Dr. ZY and Dr. FX developed the 2D version of van Herk's margin program and performed all the calculation. Dr. YC helped to collect data and participated in the research design. Dr. YC, Dr. HQ and Dr. ZY drafted the manuscript. All authors read and approved the final manuscript.

\section{Funding}

National Natural Science Foundation of China (Grant No. 81803047). Fundamental Research Funds for the Central Universities of China (Grant No. 2019kfyXKJC061).

Availability of data and materials

Please contact author for data requests.

Ethics approval and consent to participate

All patients in this study were enrolled in an institutional review board of Tongji Medical College, Huazhong University of Science and Technologyapproved retrospective data collection protocol.

\section{Consent for publication \\ Not applicable.}

Competing interests

The authors declare that they have no competing interests.

\section{Appendix}

The relationship of the margin volume and the projection area of the target.

The total projection areas $\left(\mathrm{S}_{\mathrm{YOZ}}\right)$ of the target volume in a beam direction from $0^{\circ}$ to $359^{\circ}$ were calculated. In this research, CTV is the target. The margin volume of the convention PTV margin recipe will be:

$$
V_{P T V}=\int_{0}^{\mathrm{x}_{0}+2 m} \int_{0}^{\mathrm{y}_{0}+2 m} \int_{0}^{\mathrm{z}_{0}+2 m} d x d y d z
$$

where $\mathrm{m}$ is the length of the margin, $V_{P T V}$ is the volume of PTV.

The margin volume of the BSPTV margin recipe will be:

$$
V_{B S P T V}=\int_{0}^{\mathrm{x}_{0}} \int_{0}^{\mathrm{y}_{0}+2 m} \int_{0}^{\mathrm{z}_{0}+2 m} d x d y d z
$$

where $\mathrm{m}$ is the length of the margin, $V_{B S P T V}$ is the volume of BSPTV.

The volume differences between the two margin recipes are as following:

$$
\begin{aligned}
\Delta V= & V_{P T V}-V_{B S P T V}=\int_{0}^{\mathrm{x}_{0}+2 m} \int_{0}^{\mathrm{y}_{0}+2 m} \int_{0}^{\mathrm{z}_{0}+2 m} d x d y d z \\
& -\int_{0}^{\mathrm{x}_{0}} \int_{0}^{\mathrm{y}_{0}+2 m} \int_{0}^{\mathrm{z}_{0}+2 m} d x d y d z \\
= & \int_{0}^{2 m} \int_{0}^{\mathrm{y}_{0}+2 m} \int_{0}^{\mathrm{z}_{0}+2 m} d x d y d z
\end{aligned}
$$

$\Delta V$ is the volume differences between the two margin recipes. 
If we need to spare more normal tissues, the $\Delta V$ needs to be the maximum. Hence, $\int_{0}^{\mathrm{y}_{0}+2 m} \int_{0}^{\mathrm{z}_{0}+2 m} d y d z$ needs to be the maximum. Since the $\int_{0}^{\mathrm{y}_{0}+2 m} \int_{0}^{\mathrm{z}_{0}+2 m} d y d z$ is in direct proportion to $\mathrm{S}_{\mathrm{YOZ}}$, the beam angle for the maximum of $\mathrm{S}_{\mathrm{YOZ}}$ might be chose as the best beam angle to sparing the surrounding normal tissues.

Without loss of generality, we took the target as an ellipsoid with the lengths of axis (a $(\mathrm{X}$ axis $)>b(\mathrm{Y}$ axis $)>c$ ( $\mathrm{Z}$ axis)) as shown in Fig. 4 for example. The margin volume of the ellipsoid using the convention PTV margin recipe will be:

$$
\begin{aligned}
V_{P T V-e l l i p s o i d} & =\int_{0}^{\mathrm{x}_{0}+2 m} \int_{0}^{\mathrm{y}_{0}+2 m} \int_{0}^{\mathrm{z}_{0}+2 m} d x d y d z \\
& =\frac{4}{3} \pi(a+2 m) \cdot(b+2 m) \cdot(c+2 m)
\end{aligned}
$$

where $\mathrm{m}$ is the length of the margin, $V_{P T V \text {-ellipsoid }}$ is the margin volume of the ellipsoid using the convention PTV margin recipe

$$
\begin{aligned}
V_{B S P T V-e l l i p s o i d} & =\int_{0}^{\mathrm{x}_{0}} \int_{0}^{\mathrm{y}_{0}+2 m} \int_{0}^{\mathrm{z}_{0}+2 m} d x d y d z \\
& =\frac{4}{3} \pi(c+2 m) \int_{0}^{\mathrm{x}_{0}} \int_{0}^{\mathrm{y}_{0}+2 m} d x d y
\end{aligned}
$$

where $\mathrm{m}$ is the length of the margin, $V_{\text {BSPTV-ellipsoid }}$ is the margin volume of the ellipsoid using the BSPTV margin recipe. The maximum of $V_{B S P T V \text {-ellipsoid is }}$ $\frac{4}{3} \pi a \cdot(b+2 m) \cdot(c+2 m)$ with the beam angle paraell to the $\mathrm{X}$ axis. The minimum of $V_{B S P T V \text {-ellipsoid }}$ is $\frac{4}{3} \pi(a+2 m) \cdot b \cdot(c+2 m)$ with the beam angle paraell to the $\mathrm{Y}$ axis.

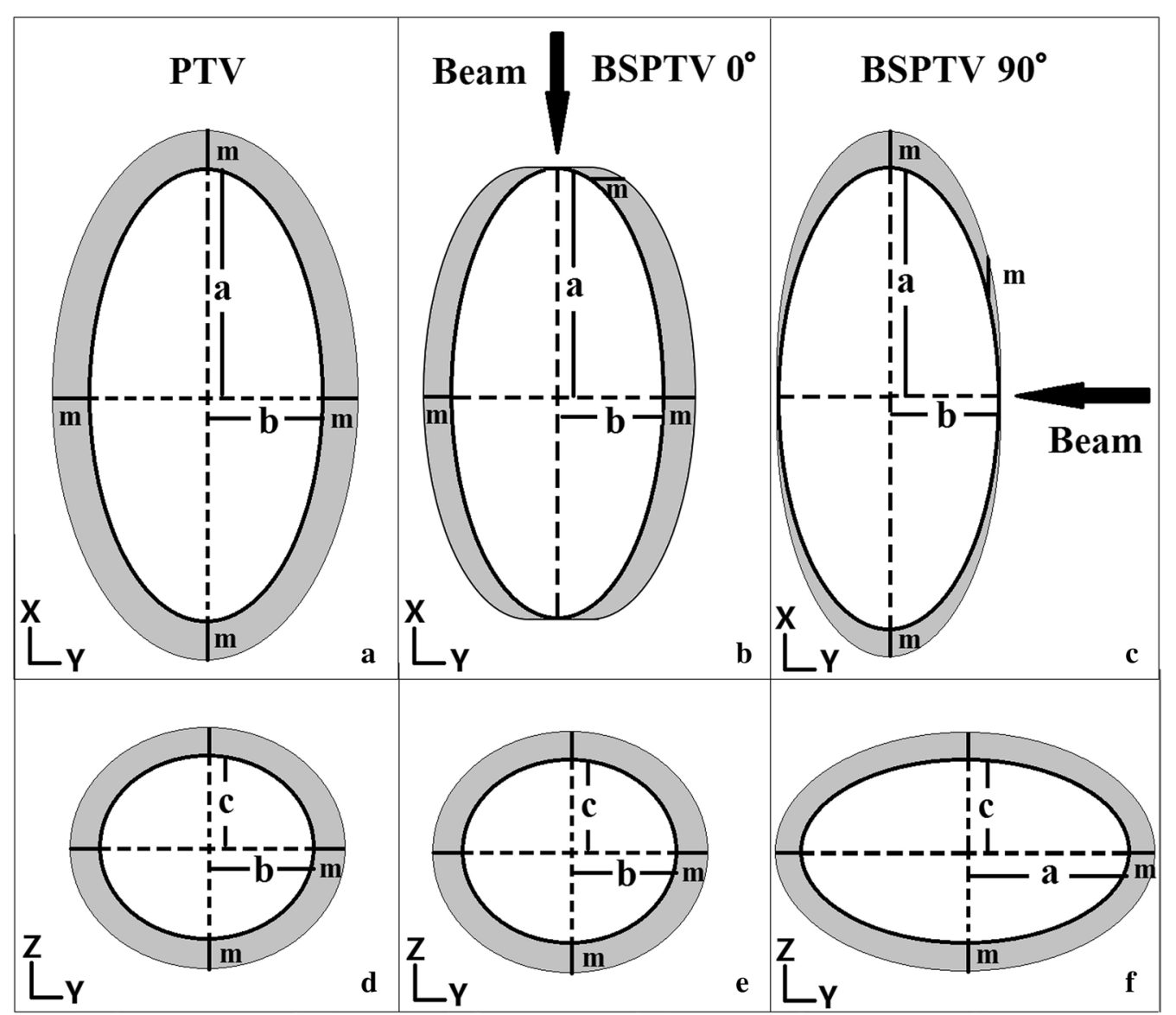

Fig. 4 The projection area of the ellipsoid CTV and the general PTV in the XOY plane and YOZ plane are shown in a, c. The CTV (thick black line) is margined isotropically with $m$ (gray area) in three directions to PTV (thin black line). The projection areas of the ellipsoid CTV and BSPTV of beam $0^{\circ}$ in the XOY plane and YOZ plane are shown in $\mathbf{b}$, $\mathbf{d}$. The margin area (gray area) of BSPTV of beam $0^{\circ}$ is smaller than that of the general PTV. The projection areas of the ellipsoid CTV and BSPTV of beam $90^{\circ}$ in the XOY plane and YOZ plane are shown in c, e. The margin area (gray area) of BSPTV of beam $90^{\circ}$ is smaller than that of both the general PTV and BSPTV of beam $0^{\circ}$ 
The volume differences between the two margin recipes are as following:

$$
\begin{aligned}
\Delta V_{\text {ellipsoid }} & =V_{P T V-\text { ellipsoid }}-V_{B S P T V-\text { ellipsoid }} \\
& =2 m \cdot \int_{0}^{\mathrm{y}_{0}+2 m} \int_{0}^{\mathrm{z}_{0}+2 m} d y d z \\
& =2 m \cdot S_{Y O Z(m \arg \text { in })}
\end{aligned}
$$

$\Delta V_{\text {ellipsoid }}$ is the volume differences between the two margin recipes.

In order to obtain the maximum $\Delta V_{\text {ellipsoid, }}$ the $\mathrm{S}_{\mathrm{YOZ}}$ needs to be the maximum as following:

$$
\begin{aligned}
& S_{Y O Z(m \arg i n)} \stackrel{\Delta \max }{=} \pi(a+2 m) \cdot(c+2 m) \\
& \Delta V_{\text {ellipsoid }}=V_{\text {ellipsoid }}-V_{\text {BSellipsoid }} \\
& =2 m \cdot \int_{0}^{\mathrm{y}_{0}+2 m} \int_{0}^{\mathrm{z}_{0}+2 m} d y d z \\
& =2 m \cdot S_{Y O Z(m \arg i n)} \stackrel{\Delta \max }{=} \frac{8}{3} m \pi(a+2 m) \cdot(c+2 m)
\end{aligned}
$$

where the beam angle for the maximum of $\mathrm{S}_{\mathrm{YOZ}}$ is parallel to the $\mathrm{Y}$ axis and perpendicular to the $\mathrm{X}$ axis, the $\Delta V_{\text {ellipsoid }}$ is maximum and the $V_{\text {BSellipsoid }}$ is the smallest. As shown in Fig. 4c, the beam angle for the maximum of $\mathrm{S}_{\mathrm{YOZ}}$ is $90^{\circ}$ in this ellipsoid case, and the beam angle for the mimmum of $\mathrm{S}_{\mathrm{YOZ}}$ is $0^{\circ}$ in this ellipsoid case.

Generalize to the general case, the value of $\mathrm{S}_{\mathrm{YOZ}}$ is calculated as the product of the average length of target in the $\mathrm{Z}$ axis and the average length of target in the XOY plane. Since the beam is irradiated perpendicular to the $Z$ axis, the average length of target in the $\mathrm{Z}$ axis is relatively a constant. Hence, the average length of target in the $\mathrm{XOY}$ plane will determine the value of $\mathrm{S}_{\mathrm{YOZ}}$. The smallest volume of BSPTV is obtained with the beam irradiated perpendicular to the maximum average length of the target in the XOY plane.

Received: 14 July 2020 Accepted: 7 October 2020

Published online: 17 October 2020

\section{References}

1. Yoon M, Kim D, Shin DH, Park SY, Lee SB, Kim DY, et al. Inter- and intrafractional movement-induced dose reduction of prostate target volume in proton beam treatment. Int J Radiat Oncol Biol Phys. 2008;71:1091-102.

2. Chang Y, Liu H-Y, Liang Z-W, Nie X, Yang J, Liu G, et al. Dosimetric effect of intrafraction tumor motion in lung stereotactic body radiotherapy using cyber knife static tracking system. Technol Cancer Res Treat. 2019;18:1533033819859448.

3. Yang Z, Chang Y, Brock KK, Cazoulat G, Koay EJ, Koong AC, et al. Effect of setup and inter-fraction anatomical changes on the accumulated dose in CT-guided breath-hold intensity modulated proton therapy of liver malignancies. Radiother Oncol. 2019;134:101-9.

4. van Herk M, Remeijer P, Rasch C, Lebesque JV. The probability of correct target dosage: dose-population histograms for deriving treatment margins in radiotherapy. Int J Radiat Oncol Biol Phys. 2000;47:1121-35.

5. van Herk M, Remeijer P, Lebesque JV. Inclusion of geometric uncertainties in treatment plan evaluation. Int J Radiat Oncol Biol Phys. 2002;52:1407-22.

6. McKenzie AL, Herk M, Mijnheer B. The width of margins in radiotherapy treatment plans. Phys Med Biol. 2000;45:3331-42.

7. Lin L, Kang M, Huang S, Mayer R, Thomas A, Solberg TD, et al. Beam specific planning target volumes incorporating 4DCT for pencil beam scanning proton therapy of thoracic tumors. Med Phys. 2015;42:3365.

8. Park PC, Zhu XR, Lee AK, Sahoo N, Melancon AD, Zhang L, et al. A beamspecific planning target volume (PTV) design for proton therapy to account for setup and range uncertainties. Int J Radiat Oncol Biol Phys. 2012;82:e329-36.

9. Tsang HS, Kamerling CP, Ziegenhein P, Nill S, Oelfke U. A novel probabilistic approach to generating PTV with partial voxel contributions. Phys Med Biol. 2017;62:4917-28.

10. Tsang HS, Kamerling CP, Ziegenhein P, Nill S, Oelfke U. Novel adaptive beam-dependent margins for additional OAR sparing. Phys Med Biol. 2018;63:215019.

11. Park PC, Cheung JP, Zhu XR, Lee AK, Sahoo N, Tucker SL, et al. Statistical assessment of proton treatment plans under setup and range uncertainties. Int J Radiat Oncol Biol Phys. 2013;86:1007-13.

12. Yang Z-Y, Chang Y, Liu H-Y, Liu G, Li Q. Target margin design for real-time lung tumor tracking stereotactic body radiation therapy using CyberKnife Xsight Lung Tracking System. Sci Rep. 2017;7:10826.

13. Yang Z, Zhang X, Wang X, Zhu XR, Gunn B, Frank SJ, et al. Multiple-CT optimization: an adaptive optimization method to account for anatomical changes in intensity-modulated proton therapy for head and neck cancers. Radiother Oncol. 2020;142:124-32.

14. Stroom J, Gilhuijs K, Vieira S, Chen W, Salguero J, Moser E, et al. Combined recipe for clinical target volume and planning target volume margins. Int J Radiat Oncol Biol Phys. 2014;88:708-14.

15. Shusharina N, Craft D, Chen Y-L, Shih H, Bortfeld T. The clinical target distribution: a probabilistic alternative to the clinical target volume. Phys Med Biol. 2018;63:155001.

16. Witte MG, Sonke J-J, Siebers J, Deasy JO, van Herk M. Beyond the margin recipe: the probability of correct target dosage and tumor control in the presence of a dose limiting structure. Phys Med Biol. 2017;62:7874-88.

17. Watkins WT, Nourzadeh $\mathrm{H}$, Siebers JV. Dose escalation in the definite target volume. Med Phys. 2020;47:3174-83.

\section{Publisher's Note}

Springer Nature remains neutral with regard to jurisdictional claims in published maps and institutional affiliations. 